\title{
The Distance to NGC 5128 (Centaurus A)
}

\author{
Gretchen L. H. Harris ${ }^{\mathrm{A}, \mathrm{D}}$, Marina Rejkuba ${ }^{\mathrm{B}}$, and William E. Harris ${ }^{\mathrm{C}}$ \\ A Department of Physics and Astronomy, University of Waterloo, Waterloo ON, N2L 3G1, Canada \\ ${ }^{B}$ European Southern Observatory, Karl-Schwarzschild-Strasse 2, D-85748 Garching, Germany \\ ${ }^{\mathrm{C}}$ Department of Physics and Astronomy, McMaster University, Hamilton ON, L8P 2X7, Canada \\ D Corresponding author. Email: glharris@astro.uwaterloo.ca
}

Received 2009 September 24, accepted 2010 February 26

\begin{abstract}
In this paper we review the various high precision methods that are now available to determine the distance to NGC 5128. These methods include: Cepheids, TRGB (tip of the red giant branch), PNLF (planetary nebula luminosity function), SBF (surface brightness fluctuations), and Long Period Variable (LPV) Mira stars. From an evaluation of these methods and their uncertainties, we derive a best-estimate distance of $3.8 \pm 0.1 \mathrm{Mpc}$ to NGC 5128 and find that this mean is now well supported by the current data. We also discuss the role of NGC 5128 more generally for the extragalactic distance scale as a testbed for the most direct possible comparison among these key methods.
\end{abstract}

Keywords: Galaxies: distances and redshifts — galaxies: individual (NGC 5128) — galaxies: stellar content

\section{Introduction}

An issue arising frequently in the literature on NGC 5128 is a lack of consensus about the best distance to adopt for this important nearby elliptical galaxy. As with any other galaxy, the assumed distance directly affects the astrophysics of all its components through the calculation of every intrinsic scale length and every luminosity at every wavelength. Some researchers use a distance of $3.9 \mathrm{Mpc}$ from a weighted average of several methods that measure the properties of old resolved stars (Rejkuba 2004). Others quote a value of $d=3.4 \mathrm{Mpc}$, which is based only on Cepheids (Ferrarese 2007). This uncomfortable uncertainty of almost $20 \%$ is much larger than one would like for a galaxy we all agree is a keystone object regardless of the wavelength region in which we work. Clearly the need exists for some kind of 'best estimate' distance.

Fortunately, there are now several distance indicators available to us, and the time is right to construct a useful mean distance measurement. None of these methods on its own can be considered by definition to give the correct value, because each is limited by its own set of systematic (and random) uncertainties. The strategic advantage of combining several methods is that it will allow us to use many different stellar components of the galaxy, each with its distinctive merits and each at least partly independent of the others.

\section{Evaluation of Five Methods}

At present we have five 'standard candles' for NGC 5128 that refer directly to properties of its stars in various ways. These methods include: Cepheids; the magnitude of the tip of the red giant branch of the oldest halo stars (TRGB); the planetary nebula luminosity function (PNLF); surface brightness fluctuations (SBF); and long period variables (LPV/Miras). Below we briefly describe these methods to give a sense of some of the issues involved and summarize the recent history of the subject.

A brief statement of the philosophical approach we will take in the discussion is appropriate at this point. In principle, we would expect each of these methods to give us the same distance to NGC 5128 within their measurement uncertainties. If those methods agree, then our confidence in the result is high. But, that confidence will be stronger if the methods are independent of each other, i.e. if they have different astrophysical underpinnings and different means of calibrating their zero points. For instance, if we find the same distance based on two methods with the same calibration base, then what we mostly know is that both methods have been applied self-consistently and appropriately. On the other hand, good agreement between two independent methods tells us more than that those methods are reliable. An important side benefit is that it also increases our confidence in cases where only one of them can be used in another galaxy. Conversely, disagreement tells us that the astrophysical or calibration foundation of one or the other, or both, needs reexamination.

In several previous papers on the extragalactic distance scale there was a tendency to normalize all calibrations to the prominent Cepheid method. But such an approach loses sight of the different strengths of the other methods and places reliance of the local distance scale too strongly on one method, creating a kind of distance-scale monoculture. Our view is that the use of multiple methods that are as independent as possible is the best way to proceed. 
NGC 5128 provides a testbed for five different methods, four of which rely on observations of resolved stars. In future it may be possible to add other stellar standard candles to the list, including RR Lyrae variables, RGB clump magnitudes, blue supergiants, Population II Cepheids, novae, or eclipsing binaries. But, at present, no data of sufficient quality for distance determination exist for such objects within NGC 5128 .

\subsection{Cepheids}

Distances based on Cepheids use the well-known relation between pulsation period and luminosity (Leavitt law; Leavitt \& Pickering 1912) to infer the luminosity of Population I Cepheid variable stars. One of the major results from the Hubble Space Telescope (HST), and certainly among the most well known, is the determination of the Hubble constant in the $H_{0}$ Key Project (Kennicutt, Freedman \& Mould 1995; Freedman et al. 2001). As described by Ferrarese et al. (2000) this project depended heavily on Cepheids, and was designed to establish Cepheids as the primary standard candle. The great success of the Key Project and the attention paid to it resulted also in wide acceptance of Cepheids as reliable distance indicators.

However, in the last decade, the number of papers discussing the influence of chemical composition, and on-going debate on dependence or independence of the Cepheid period-luminosity (PL) relation on metallicity (with discrepant results even about the sign of the dependence on iron abundance) continues to be high, culminating in a recent claim by Romaniello et al. (2008) that the Cepheid PL relation is not universal. In addition there are other discussions and criticisms (some rejected) of biases due to blending in distant crowded extragalactic fields, the longest observable period, dust extinction, or cut-off due to limiting absolute magnitude of the Cepheid sample (e.g. Bresolin et al. 2005; Paturel \& Teerikorpi 2005; Tammann et al. 2008). The successful comparison of Cepheids with other standard candles (e.g. Rizzi et al. 2007; Mould \& Sakai 2009) and numerous Cepheid distance scale calibrations with a very wide range of methods, such as theoretical non-linear pulsation models, parallaxes (from both Hipparcos and from HST), the Baade-Wesselink method, the water maser distance to NGC 4258, and main sequence fitting, certainly confirms that Cepheids are good distances indicators. But the above mentioned limitations and the possibility of a nonuniversal PL relation should warn the reader that Cepheids are not flawless standard candles and - as for any other standard candle - the quoted distances have to be taken into account with their error-bars included.

As already mentioned, the primary uncertainties in Cepheid distances are reddening and the dependence of the PL relation on chemical abundance. Both factors are important in this case. The NGC 5128 Cepheids are found in and near the central dust lane because Cepheids are relatively young stars and would not be present in the old (gas poor) stellar halo of an E galaxy. Their individual reddenings are large, ranging from $E(V-I) \simeq 0.4$ to 0.8 and, combined with somewhat uncertain values for the ratio of total to selective absorption, can lead to larger uncertainties in the dereddened absolute magnitudes. In addition, NGC 5128 is the first elliptical galaxy in which Cepheids have been identified, making the environment somewhat unusual. Ferrarese et al. (2007) quote a Cepheid distance modulus of $(m-M)_{0}=27.67 \pm 0.12 / 0.16$; the first (smaller) uncertainty is random and the second is systematic. For straightforward comparison with other methods, we have added these in quadrature to give a net uncertainty of \pm 0.2 . This distance is based on a calibration of the Cepheid PL relation in the LMC, and assumes an LMC distance modulus of $(m-M)_{0}=18.50$ (Freedman et al. 2001), the widely agreed-on contemporary value (Schaefer 2008).

\section{$2.2 T R G B$}

The red giant branch tip method relies on observationally and theoretically well understood characteristics of Population II stars, which are the most abundant stars in an elliptical galaxy. The maximum luminosity (tip) of the red giant branch represents the core helium ignition stage of low-mass stars, which happens at approximately the same core mass. For ages in the range $\sim 2-15$ Gyr this implies that the bolometric luminosity of the tip for stars with the same metallicity varies by at most $0.1 \mathrm{mag}$, resulting in a sharp discontinuity in the luminosity function (LF) of the red giant branch at that point. This discontinuity in the LF was established in globular clusters and dwarf galaxies by Da Costa \& Armandroff (1990) and Lee et al. (1993), who provided the first calibration and detection method for the TRGB as distance indicator. In fact, these papers represent the modern re-definition of a standard candle that was used long ago for globular clusters in the Milky Way (the mean luminosity of the brightest giants; see, for example, Shapley 1918) and was a key element in establishing the size and structure of the Galaxy.

When used in objects containing a significant population of old and relatively metal-poor stars, TRGB is a clean and powerful standard candle. Observationally it relies only on obtaining single-epoch accurate photometry of a large sample of stars in a halo field. Another advantage is that reddening or differential reddening for halo stars is not usually a significant problem in the analysis, as opposed to Cepheids, which are typically located in star forming regions with patchy extinction. See Rizzi et al. (2007) for a valuable and more extensive summary of the characteristics of the method.

The TRGB method can be used in either optical or near-infrared wavebands, but is most widely established in the $I$-band. In the $I$-band, the metallicity dependence of the RGB tip luminosity is small: in particular, for stars with metallicity below $[\mathrm{Fe} / \mathrm{H}]=-0.7$, the TRGB is essentially 'flat' in $M_{I}$ and has been well calibrated both theoretically and experimentally through a combination of stellar evolution models and the data for the nearby extremely rich Milky Way globular cluster $\omega$ Centauri. Bellazzini et al. (2001) have refined the model-based 
calibration of this technique, obtaining $M_{I}^{T R G B}=-4.04 \pm$ 0.12 for stars in this metal-poor range. An additional thorough update of the calibration (Rizzi et al. 2007) which uses a combination of RGB data in Local Group dwarf galaxies, finds $M_{I}^{T R G B}=-4.05 \pm 0.10$ for this metal-poor side of the RGB tip. In practice, this means that for any galaxy or star cluster whose stellar population has a strong metal-poor component, the RGB tip will be resolved at this luminosity level. Since the halo stars of NGC 5128 cover the entire metallicity range from $[\mathrm{Fe} / \mathrm{H}] \simeq-2.0$ up to above Solar abundance (Rejkuba et al. 2005), this condition is easily satisfied. Thus the simplest application of the method is to determine the tip magnitude at the metal-poor side in $I$ where it first resolves, and subtract the absolute magnitude.

$(V, I)$ photometry of resolved stars in three halo fields (at projected galactocentric distances of $\sim 20,30$, and $40 \mathrm{kpc}$ ) has been done in NGC 5128 with the HST cameras WFPC2 and ACS/WFC (Harris et al. 1999; Harris \& Harris 2000, 2002; Rejkuba et al. 2005). The results for the 40-kpc field, which is the deepest of the three, are shown in Figure 1. We have redone the TRGB calculations for all three fields using the smoothed probability density function LF methods described in those papers, and find $I^{T R G B}=24.10 \pm 0.10$ for each of these outer-halo fields at the blue, metal-poor side of the colour-magnitude diagram (CMD). Combined with the most recent Rizzi et al. (2007) calibration and using $A_{I}=0.22 \pm 0.02$ (Schlegel et al. 1998), with reddening law from Cardelli et al. (1989), we therefore find $(m-M)_{0}=27.93 \pm 0.13$ for the average of the three regions.

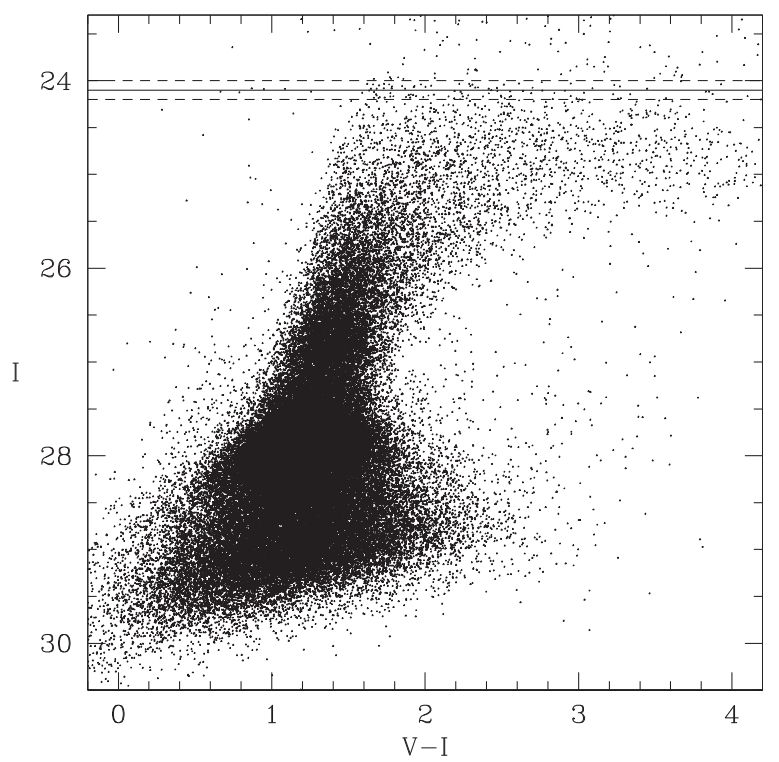

Figure 1 Color-magnitude diagram for the outer halo of NGC 5128, from Rejkuba et al. (2005). The photometry is from HST ACS/ WFC imaging in $V$ and $I$ and includes roughly 70000 stars at a location $40 \mathrm{kpc}$ from the galaxy center. The horizontal, solid line at $I=24.1$ shows the deduced luminosity of the TRGB, with dashed lines showing the measurement uncertainty of \pm 0.1 mag.
Rizzi et al. (2007) provide a thorough discussion of the TRGB zero point and application of the method to several nearby galaxies. As part of their analysis they derive several empirical equations that describe the dependence of the zero point of the TRGB luminosity on the metallicity (or color) of the stellar population in various filters including $(I, J, H, K)$ and the HST flight system F814W. Their analysis confirms that in $I$ or F814W the metallicity dependence of the tip luminosity is quite modest, and virtually flat for $[\mathrm{Fe} / \mathrm{H}]<-1.2$. They also remeasured the tip magnitude for NGC 5128, finding $I^{T R G B}=24.03 \pm 0.02$ (internal uncertainty). This estimate nominally agrees well with ours within the uncertainties of both. However, they appear to have used the raw WFPC2 data for the inner-halo $8 \mathrm{kpc}$ field (Harris \& Harris 2002), which is by far the most affected by crowding and the least suitable of the four available halo pointings in NGC 5128. The much deeper and cleaner ACS data, covering a wider field of view (see Figure 1), give a considerably sharper definition of the tip magnitude, as do the two other outer-halo fields that we have used here. In addition, Rizzi et al. (2007) apply $M_{I}^{T R G B}=-3.90$, a value more appropriate for the red, metal-rich side of the RGB tip rather than the metal-poor side, which represents the true onset of the RGB tip in any optical bandpass.

Madore et al. (2009) discuss in detail the modified detection method for measuring the luminosity of the TRGB in composite stellar populations (such as galaxy halos) accounting explicitly for the metallicity (or color) dependence of the slope of the tip magnitude. In this new method the impact of reddening is further reduced.

While much less used in the near-infrared, TRGB has the advantage of an even more reduced dependence on reddening. However, the larger dependence of the tip magnitude on metallicity in $J, H$ and $K$-bands with respect to $I$ (see also Figure 2 of Rizzi et al. (2007)) has made this method less popular in the near-infrared. Using deep JHKs ground-based observations of a halo field at a projected distance of $18 \mathrm{kpc}$ north-east from the center, Rejkuba (2004) found $(m-M)_{0}=27.89 \pm$ $0.20(\mathrm{~J}), 27.90 \pm 0.20(\mathrm{H})$, and $27.88 \pm 0.16(\mathrm{~K})$. The calibration adopted for the $K s$ band is based on an empirical relation of RGB tip magnitude as a function of metallicity for Galactic globular clusters (Ferraro et al. 2000), which is in agreement with the most recent and first geometric calibration of the TRGB absolute magnitude at $M_{K}=-6.85 \pm 0.03$ based on Hipparcos parallaxes of Solar-neighborhood Galactic red giants (Tabur et al. 2009). The $J$ and $H$-band calibrations have somewhat larger uncertainty and are based primarily on stellar evolutionary models (Bertelli et al. 1994) and an estimate of the Galactic Bulge TRGB magnitude (Zoccali et al. 2003). A simple weighted average of these three near-infrared TRGB distance moduli gives $(m-M)_{0}=27.89 \pm 0.11$.

Averaging together the optical and near-infrared results, we obtain $(m-M)_{0}(T R G B)=27.91 \pm 0.08$. 


\subsection{PNLF}

It was shown in the late 1970 s that the PNLF is an excellent standard candle for distance measurement to nearby galaxies (see e.g. Ciardullo et al. 2002; Ciardullo 2003, for a comprehensive recent discussion). Logistically similar to the TRGB method, it employs intermediate-age and old stellar populations and relies on the sharply defined upper end of the PNLF, $M^{\star}$, as measured in the [OIII] emission line. Technically, the PNLF distance is derived by fitting the observed LF to an empirical law, and calibrating the observed magnitude with respect to PNLF cutoff magnitude in M31. The PNLF method is well calibrated within the Local Group and has been shown to be consistent in different group and galaxy environments. It is also particularly helpful in bridging the Population I and II distance scale techniques, since $\mathrm{PNe}$ can be commonly found in both spiral and elliptical galaxies (Ciardullo et al. 2002).

Hui et al. (1993) determined the PNLF for 785 planetary nebulae in NGC 5128 and found a distance by matching with the PNLF data in M31 and using the then-standard fiducial M31 distance of $710 \mathrm{kpc}\left((m-M)_{0}=24.26\right)$. Combining the uncertainties due to the M31 distance, the PNLF model, and the filter calibration they quote a final best-estimate distance modulus for NGC 5128 of $(m-M)_{0}=27.73 \pm$ 0.14 . However, the current nominal value for the distance to $\mathrm{M} 31$ is $775 \mathrm{kpc}\left((m-M)_{0}=24.45 \pm 0.07\right)$, which is based on the Cepheid distance modulus (Ferrarese et al. 2000), the more recent TRGB value (McConnachie et al. 2005), and RR Lyraes (Brown et al. 2004; Sarajedini et al. 2009), all of which now agree to within 0.1 magnitude. This revision brightens the fiducial PNLF luminosity to $M^{\star}=-4.67 \pm 0.07$. From the numbers in Hui et al. (1993) we find that the apparent magnitude of the PNLF tip is $m^{\star}=23.25 \pm 0.07$ and the resulting distance modulus to NGC 5128 is then $(m-M)_{0}=27.92 \pm 0.12$.

\subsection{Mira Variables (LPV)}

Miras are large amplitude, long-period variables (LPVs) at the tip of the asymptotic giant branch (AGB). The classic long-period Mira variable stars, with periods shorter than $\sim 400$ days, follow a well defined infrared and bolometric PL relation (Feast \& Whitelock 1999; Whitelock et al. 2008). The Mira PL relation was first found from statistical parallax work, but only with observations in near-infrared did it become clear that the scatter of this relation in the $K$-band (and in bolometric magnitude) is small enough to be useful for distance determination to other galaxies $\left(\sigma_{K}=0.13\right.$ and $\sigma_{M b o l}=0.16$, Feast \& Whitelock 1999). The latest calibration of the Mira PL relation is based on the analysis of the Hipparcos data for large-amplitude AGB variables in the Milky Way (for detailed discussion see Whitelock et al. 2008). Other calibrations that reinforce the definition of the PL relation use the Milky Way globular clusters and the LMC Miras. These additional data therefore rely on RR Lyrae and subdwarf main-sequence-fitting distances for globular clusters, and on the variety of the sources for the LMC distance moduli.

This method is strongly reminiscent of the Cepheid method, and like the Cepheids, the relation is well calibrated from within the Local Group (as noted above, primarily from the Milky Way and LMC). Another similarity with Cepheids is the possible (but in the $K$-band small and debated) metallicity dependence of the PL zero point (Feast 2004). On the other hand, use of the infrared photometry minimizes errors due to uncertain extinction (and reddening law).

Rejkuba (2004) measured several hundred long-period variable stars to construct a PL relation for the Miras in two halo fields of NGC 5128. The slope of the relation is the same as for Miras in the LMC and the Milky Way to well within the internal uncertainties of all. The zero point of the PL relation is taken from the LMC for which (as above for the Cepheids $) d \equiv 50 \mathrm{kpc}\left((m-M)_{0}=18.50\right)$. The resulting distance modulus for NGC 5128 based on Miras quoted by Rejkuba (2004) is $(m-M)_{0}=27.96 \pm 0.11$.

\section{$2.5 S B F$}

The surface brightness fluctuations method, pioneered by Tonry and collaborators in the late 1980 s, uses the spatial fluctuation signal in the smooth integrated light of the brightest component of the stellar population to determine the distance to its parent galaxy (see Tonry et al. 2001 and references therein for discussion of the method). In an old stellar population typical for elliptical galaxies, measurements in the $I$-band are dominated by the red giant branch stars, and while the absolute fluctuation magnitude is expected to vary with age and metallicity of a stellar population, its mean color can be used to constrain the distance measurements to $\sim 10 \%$ accuracy (Blakeslee et al. 2009). Tonry et al. (2001) quoted $(m-M)_{0}=28.12 \pm 0.14$ for NGC 5128. The SBF method is best applied to galaxies with smooth bulge light dominated by an old stellar population ( $\mathrm{S} 0$ and ellipticals), but its calibration has so far relied heavily on Cepheids. NGC 5128 is actually the first (and only) elliptical galaxy where both methods may be applied. So far, the calibration from Cepheids has used either the association between SBF distances to spiral galaxy bulges for those spiral galaxies that have Cepheid distances, or $\mathrm{SBF}$ distances to ellipticals and S0 galaxies in groups that host spirals with Cepheid distances (Tonry et al. 2000; Blakeslee et al. 2002). Efforts to improve the understanding of the SBF magnitudes as a function of stellar population, and SBF measurements in Magellanic Cloud clusters, have led to re-calibrations of the SBF zero point.

An inherent uncertainty of the SBF technique is that it requires knowledge of the mean colour of the underlying population to account for metallicity and stellar population differences. In the case of NGC 5128 this is more difficult to determine because it is so nearby and spread out across the sky. Additionally, differences in internal reddening and an age mixture of populations may be present. These difficulties are emphasized as well by Ferrarese et al. (2007). New optical wide-field observations 
of the galaxy (Peng et al. 2004), combined with the updated zero point of the SBF distance scale, led to the revised SBF distance to NGC 5128 of $(m-M)_{0}=27.74 \pm 0.14$, reported by Ferrarese et al. (2007). We adopt here this SBF distance value.

The SBF method requires additional comment because it is the only one that is difficult to calibrate from Local Group members alone. The other four can be thought of as 'primary' techniques in the sense that they use the properties of directly resolved individual stars and ultimately rest on well understood stellar physics: the Miras and Cepheids both have well defined PL relations (both with calibrations made with parallax methods), while the TRGB and PNLF use the fiducial luminosity at a breakpoint along the evolutionary path of old stars. SBF is a 'secondary' method in the sense that it relies on the integrated light of an ensemble of stars and must use the primary methods to set its zero point. Recently the SBF zero point now has a calibration using TRGB distance measurements (Mould \& Sakai 2009).

Lastly, TRGB and SBF both use the same fundamental stellar population — the old RGB stars — and thus in principle they should give the same distances for the same galaxy. The main difference between them is, again, that TRGB uses the resolved stars at the bright tip of the red giant branch, while SBF measures the mean luminosity of the entire unresolved or partially resolved RGB population. NGC 5128 is one of a very small number of galaxies beyond the Local Group in which we can make a direct comparison between the two. As can be seen from the summary table, although they agree formally (within the quoted uncertainties), the resulting distances differ by $0.3 \mathrm{Mpc}$ or almost $10 \%$.

We can make a similar comparison of TRGB and SBF distances for the Leo Group ellipticals NGC 3377 and NGC 3379, which at $d \sim 10 \mathrm{Mpc}$ are close enough that HST/ACS imaging can resolve their brightest halo stars. For NGC 3377, Harris et al. (2007a) find a TRGB distance modulus of $30.18 \pm 0.16$, which is remarkably close to the SBF value of $30.19 \pm 0.09$ given in Tonry et al. (2001). The agreement in NGC 3379 is nearly as good where Harris et al. (2007b) find $(m-M)_{0}=30.1 \pm 0.16$ for the TRGB, compared with the Tonry et al. (2001) SBF value of $30.06 \pm 0.11$. This suggests to us that the much greater difference between the NGC 5128 SBF and TRGB distances may be due to factors such as uncertainties in the colour of the underlying galaxy light as already mentioned above, or the partial presence of a younger stellar component (brighter than the RGB tip), which would make it appear brighter and therefore closer (Raimondo et al. 2005). The latter possibility warrants particular attention since the thermally pulsing AGB component (TP-AGB) is surely present in NGC 5128 as testified by Mira LPVs (Rejkuba et al. 2003).

By contrast, the TRGB luminosity is much less ambiguous since it is based on clearly resolved stars, has been determined for several NGC 5128 halo fields, and is well normalized for metallicity. Thus NGC 5128 is a galaxy for which the TRGB distance can be argued to supersede the SBF distance.

\section{Discussion and Conclusions}

The extragalactic distance scale is a classic astronomical subject whose roots extend far into the past. It will already be evident from the discussion in preceding sections that the calibrations and zero points of standard candles are a constant source of concern, revision, and debate in the distance scale literature. With the appearance of new distance calibrators, several specialist conferences have been dedicated to this topic in the last two decades. Among the newcomers in the group of well established distance candles over the last $\sim 20$ years are TRGB, PNLF, and SBF, three of the five distance indicators used to determine the distance to NGC 5128.

Although we have stressed the advantages of combining independent approaches, none of our four key resolved-star standard candles can ultimately be said to be totally independent of all the others. The distance scale for the nearby universe requires the careful assembly and intercomparison of many techniques, with checks and balances at every outward step. Convergence may at times seem remarkably slow and difficult. Nevertheless, at this stage in the development of the subject, wide agreement to within $\pm 0.1 \mathrm{mag}$ has been achieved for galaxies within the Local Group. We use this 'near-field' Local Group region as our starting point.

Brief comparisons of the basis of each method show how they are interrelated at their starting points and how none is truly 'independent' of the others. But these same points of overlap provide several strong consistency checks:

1. The fundamental calibration of the Cepheid PL relation relies on trigonometric parallaxes of nearby Cepheids and main-sequence fitting to young Milky Way star clusters containing Cepheids, often supplemented by Baade-Wesselink method parallaxes and the PL slope from the LMC (for only a recent sampling of the vast literature, see van Leeuwen et al. 2007; An et al. 2007; Groenewegen 2008).

2. The TRGB luminosity calibration (well reviewed by Rizzi et al. 2007) relies on observations of the tip luminosity in Milky Way globular clusters (particularly $\omega$ Centauri) and the old-halo components of Local Group dwarf galaxies, all of which distances rely in turn on the luminosities of the old RR Lyrae and horizontal-branch stars.

3. The calibration of the Mira PL relation rests on local Milky Way long-period variables and importantly on the LMC LPVs; the LMC fiducial distance relies in turn on a mixture of indicators including RR Lyraes, Population I and II Cepheids, and the expansion-shell parallax to SN1987a.

4. Finally, the PNLF calibration that we adopt here depends strongly on the distance to M31, which in turn is calibrated from Cepheids, Miras, RR Lyraes, and TRGB. 
Table 1. Summary of distance calibrations

\begin{tabular}{lc}
\hline Method & $(m-M)_{0}$ \\
\hline Cepheids & $27.67 \pm 0.20$ \\
TRGB (I), (JHK) & $27.91 \pm 0.08$ \\
PNLF & $27.92 \pm 0.10$ \\
LPV (Miras) & $27.96 \pm 0.11$ \\
SBF & $27.74 \pm 0.14$ \\
$\langle m-M\rangle_{0}$ & $27.91 \pm 0.05^{\mathrm{a}}$ \\
$\langle d\rangle(\mathrm{Mpc})$ & $3.82 \pm 0.09^{\mathrm{a}}$
\end{tabular}

${ }^{\mathrm{a}}$ Average of the first four methods listed (see text).

As soon as we step beyond the Local Group, measurements of distances to individual galaxies are occasionally affected by larger disagreements among the methods that are sometimes still hard to understand (see the papers above for several examples). For NGC 5128, however, the various methods have finally begun to converge, showing encouraging agreement to within their internal uncertainties. It is worth noting that the true uncertainty applicable to each method is currently dominated by the external accuracy of the calibration, which is \pm 0.1 mag or greater. The internal measurement uncertainty - e.g. the apparent magnitude of the RGB tip, or $M^{*}$ for the PNLF — is now below $0.1 \mathrm{mag}$ thanks to large observational samples and rigorous numerical analysis methods.

Table 1 summarizes our results. The uncertainty quoted for each one is the combination of internal and external errors. A simple weighted mean of all five methods gives $(m-M)_{0}=27.89 \pm 0.04$ or $d=3.77 \pm$ $0.08 \mathrm{Mpc}$. Given the discussion above, however, we recommend a final average based on the four primary, resolved-star methods (Cepheids, TRGB, PNLF, and Miras). This average gives a slightly larger distance of $(m-M)_{0}=27.91 \pm 0.08$ or $d=3.8 \pm 0.1 \mathrm{Mpc}$.

The mutual agreement among these methods is about as good as we have for any galaxy beyond the Local Group. It appears that, to within $\pm 0.1 \mathrm{Mpc}$, the recommended distance of $3.8 \mathrm{Mpc}$ for NGC 5128 is well supported by the evidence at hand.

\section{Acknowledgments}

GLHH and WEH acknowledge financial support through research grants from the Natural Sciences and Engineering Research Council of Canada. The germ of this paper was initiated during a visit to ESO Garching, sponsored through the ESO visitor programme.

\section{References}

An, D., Terndrup, D. M. \& Pinsonneault, M. H., 2007, ApJ, 671, 1640 Bellazini, M., Ferraro, F. R. \& Pancino, E., 2001, ApJ, 556, 635

Bertelli, G., Bressan, A., Chiosi, C., Fagotto, F. \& Nasi, E., 1994, A\&AS, 106, 275

Blakeslee, J. P., Lucey, J. R., Tonry, J. L., Hudson, M. J., Narayanan, V. K. \& Barris, B. J., 2002, MNRAS, 330, 443

Blakeslee, J. P. et al., 2009, ApJ, 695, 556

Bresolin, F., Pietrzyński, G., Gieren, W. \& Kudritzki, R.-P., 2005, ApJ, 634, 1020
Brown, T. M., Ferguson, H. C., Smith, E., Kimble, R. A., Sweigart, A. V., Renzini, A. \& Rich, R. M., 2004, AJ, 127, 2738

Cardelli, J. A., Clayton, G. C. \& Mathis, J. S., 1989, ApJ, 345, 245

Ciardullo, R., Feldmeier, J. J., Jacoby, G. H., Kuzio de Naray, R., Laychak, M. B. \& Durrell, P. R., 2002, ApJ, 577, 31

Ciardullo, R., 2003, IAUS 209, in 'Planetary Nebulae: Their Evolution and Role in the Universe', Eds. Kwok, S., Dopita, M. \& Sutherland, R., 617

Da Costa, G. S. \& Armandroff, T. E., 1990, AJ, 100, 162

Feast, M., 2004, in 'Variable Stars in the Local Group', IAU Colloquium 193, Eds. Kurtz, D. W. \& Pollard, K. R., ASP Conference Proceedings, Vol. 310, 304

Feast, M. \& Whitelock, P., 1999, in 'Post-Hipparcos cosmic candles', Eds. Heck, A. \& Caputo, F. (Dordrecht; Boston: Kluwer Academic Publishers) (Astrophysics and space science library; v. 237), 75

Ferrarese, L. et al., 2000, ApJ, 529, 745

Ferrarese, L., Mould, J. R., Stetson, P. B., Tonry, J. L., Blakeslee, J. P. \& Ajhar, E. A., 2007, ApJ, 654, 216

Ferraro, F. R., Montegriffo, P., Origlia, L. \& Fusi Pecci, F., 2000, AJ, 119, 1282

Freedman, W. L. et al., 2001, ApJ, 553, 47

Groenewegen, M. A. T., 2008, AAp, 488, 25

Harris, G. L. H., Harris, W. E. \& Poole, G. B., 1999, AJ, 117, 855

Harris, G. L. H. \& Harris, W. E., 2000, AJ, 120, 2423

Harris, W. E. \& Harris, G. L. H., 2002, AJ, 123, 3108

Harris, W. E., Harris, G. L. H., Layden, A. C. \& Stetson, P. B., 2007a, AJ, 134, 43

Harris, W. E., Harris, G. L. H., Layden, A. C. \& Wehner, E. M. H., 2007b, ApJ, 666, 903

Hui, X., Ford, H. C., Ciardullo, R. \& Jacoby, G. H., 1993, ApJ, 414, 563

Kennicutt, R. C., Freedman, W. L. \& Mould, J. R., 1995, AJ, 110, 1476

Leavitt, H. S. \& Pickering, E. C., 1912, Harvard College Observatory Circular, Vol. 173, 1-3

Lee, M. G., Freedman, W. L. \& Madore, B. F., 1993, ApJ, 417, 553

Madore, B. F., Mager, V. \& Freedman, W. L., 2009, ApJ, 690, 389

McConnachie, A. W., Irwin, M. J., Ferguson, A. M. N., Ibata, R. A., Lewis, G. F. \& Tanvir, N., 2005, MNRAS, 356, 979

Mould, J. \& Sakai, S., 2009, ApJ, 697, 996

Paturel, G. \& Teerikorpi, P., 2005, A\&A, 443, 883

Peng, E. W., Ford, H. C. \& Freeman, K. C., 2004, ApJS, 150, 367

Raimondo, G., Brocato, E., Cantiello, M. \& Capaccioli, M., 2005, AJ, 130, 2625

Rejkuba, M., Minniti, D., Silva, D. R. \& Bedding, T. R., 2003, A\&A, 411,351

Rejkuba, M., 2004, A\&A, 413, 903

Rejkuba, M., Greggio, L., Harris, W. E., Harris, G. L. H. \& Peng, E. W., 2005, ApJ, 631, 262

Rizzi, L. et al., 2007, ApJ, 661, 815

Romaniello, M. et al., 2008, A\&A, 488, 731

Sarajedini, A., Mancone, C. L., Lauer, T. R., Dressler, A., Freedman, W., Trager, S. C., Grillmair, C. \& Mighell, K. J., 2009, AJ, 138, 184

Schaefer, B. E., 2008, AJ, 135, 112

Shapley, H., 1918, ApJ, 48, 89

Schlegel, D. J., Finkbeiner, D. P. \& Davis, M., 1998, ApJ, 500, 525

Tammann, G., Sandage, A. \& Reindl, B., 2008, ApJ, 679, 52

Tabur, V., Kiss, L. L. \& Bedding, T. R., 2009, ApJLett, 703, 72

Tonry, J. L., Blakeslee, J. P., Ajhar, E. A. \& Dressler, A., 2000, ApJ, 530,625

Tonry, J. L., Dressler, A., Blakeslee, J. P., Ajhar, E. A., Fletcher, A. B., Luppino, G. A., Metzger, M. R. \& Moore, C. B., 2001, ApJ, 546, 681

Van Leeuwen, F., Feast, M. W., Whitelock, P. A. \& Laney, C. D., 2007, MNRAS, 379, 723

Whitelock, P. A., Feast, M. W. \& van Leeuwen, F., 2008, MNRAS, 386,313

Zoccali, M. et al., 2003, A\&A, 399, 931 